\title{
The Models of Aviation Ammunition Equipment Maintenance Materials
}

\section{Consumption}

\author{
Zhengwei $\mathrm{Li}^{1}$, Yan Liu ${ }^{2}$, Shenyang Liu ${ }^{3}$, Guangxu Luo ${ }^{3}$ \\ ${ }^{1}$ Department of Aviation Ammunition, Air Force Logistics College, Xuzhou 221000, China; \\ ${ }^{2}$ Department of Basic, Air Force Logistics College, Xuzhou 221000, China; \\ ${ }^{3}$ Department of Aviation Four Stations, Air Force Logistics College, Xuzhou 221000, China
}

Keywords: Aviation ammunition equipment, maintenance materials, consumption rule, probability theory

\begin{abstract}
The aviation ammunition equipment maintenance materials consumption rule has a great significance on all the segments about materials including acquisition, storage, supplying and management. This paper combines aviation ammunition equipment maintenance materials consumption rule under corrective maintenance with aviation ammunition equipment maintenance materials consumption rule under condition-based maintenance. Then the calculation method of aviation ammunition equipment maintenance materials reserve is presented on the basis of the aviation ammunition equipment maintenance materials consumption rule. The calculation method is used to solve the difficult problem of developing a reserve program of aviation ammunition equipment maintenance materials, since it is not easy to find out aviation ammunition equipment maintenance materials consumption rule under condition-based maintenance in the current study.
\end{abstract}

\section{INTRODUCTION}

Many scholars have made scientific researches on materials consumption prediction. Zhao Jianzhong improves on search mode of APSO and the weighted method of least squares support vector machine. Then the consumption forecasting model of missile materials is established based on RS, EW and WLS-SVM with APSO, and realization process is analyzed. The example results show that the combinatorial forecasting model has better forecast precision and important applied value in the course of consumption forecasting of missile materials Ni Xiancun uses the concept of the repair degree and improves the proportional hazards model based on general renewal process (Ni Xiancun, 2009). The parameter value is estimated by analyzing failure data and then the number rotables are calculated based on Monte Carlo simulation. An example is given and the results of various maintenance policies with and without considering covariates are compared and analyzed. Results show that the model has a larger practical value. Li Dawei uses the initial materials scheme as prior information and proposes the regulate method of materials in incipient operation based on the Bayes method (LI Dawei, 2013). Finally, the simulation example shows that the method proposed is feasibility. Comparing with classical methods, the method proposed can improve the accuracy of materials consumption estimation and has good steadiness. So the more rational materials scheme can be formed (Zhao Jianzhong, 2012).

The predictive precision of models applied to predict the aviation ammunition equipment maintenance materials consumption can be enhanced effectively by using the information of the reliability of the units and the maintenance strategy.

\section{MATERIALS CONSUMPTION RULE}

Suppose the equipment begins to work from 0 time, from which to the first condition-based maintenance with condition-based replacement unit and the time interval is $T$. Regarding the value of $T$ : if the equipment is under minor repair, condition-based maintenance with condition-based replacement units is adopted and $T$ value is the interval time for minor repair ; if non-replacement of condition-based replacement units is adopted in the situation of minor repair, as well as the condition-based maintenance is adopted under the condition of medium repair, the $T$ value is the interval time for medium repair. Major maintenance means conducting a comprehensive repair in order to achieve an all round recover of equipment properties according to technical standard, which enable the equipment to reach /get close to the standard of brand-new equipment or the specified technical performance. Normally, the equipment will be received with a comprehensive replacement of condition-based replacement units; therefore, the condition-based maintenance is not in consideration when equipment is under major repair.

Under the condition of condition-based maintenance, the aviation ammunition equipment maintenance materials consumption rule within the working time $[0,+\infty)$ can be analyzed based on reliability theory and probability theory. However, due to the complexity of the aviation ammunition equipment maintenance materials consumption rule within the equipment working time $(T,+\infty)$, in this paper only the aviation ammunition equipment maintenance materials consumption rule within $[0, T]$ is studied. Under the condition of condition-based maintenance, the derivation process of aviation ammunition equipment maintenance 
materials consumption rule within $[0, T]$ time period is shown as below.

(1) Aviation ammunition equipment maintenance materials consumption rule of corrective maintenance

During $[0, T)$, the probability of aviation ammunition equipment maintenance materials consumption $y_{1}=k$ ( $k \geq 1$ ) caused by corrective maintenance equals the different value between the probability of the service life of condition-based replacement units ( $k$ numbers) is smaller than $T$ and the probability of the service life of condition-based replacement units ( $k+1$ numbers) is smaller than $T$ :

$$
P\left(y_{1}=k\right)=P\left(\sum_{i=1}^{k} T_{i}<T\right)-P\left(\sum_{i=1}^{k+1} T_{i}<T\right)
$$

During $[0, T)$, the probability of aviation ammunition equipment maintenance materials consumption $y_{1}=0$ caused by corrective maintenance equals the probability of the service life of the first condition-based replacement unit is bigger than $T$, which is

$$
P\left(y_{1}=0\right)=P\left(T_{1}>T\right)
$$

During $[0, T)$, the average value of aviation ammunition equipment maintenance materials consumption caused by corrective maintenance is

$$
\bar{y}_{1}=\sum_{k=0}^{+\infty} k P\left(y_{1}=k\right)=\sum_{k=1}^{+\infty} k\left[P\left(\sum_{i=1}^{k} T_{i}<T\right)-P\left(\sum_{i=1}^{k+1} T_{i}<T\right)\right]
$$

(2) Aviation ammunition equipment maintenance materials consumption rule of condition-based maintenance

Suppose the rated value for a replacing conditionbased replacement unit is $w$, which means under condition-base maintenance, if the reliability of a condition-based replacement unit falls below $w$, replacing maintenance will be conducted. Suppose $R\left(T_{0}\right)=w$, so $T_{0}=R^{-1}(w)$, which means when conditionbased maintenance is conducted at $T$ time, if the working time of a unit exceeds $T_{0}\left(T_{0}<T\right)$ under condition-based replacement, replacing maintenance will be conducted, otherwise no replacement will be given.

It is not difficult to prove that the probability for aviation ammunition equipment maintenance materials consumption $y_{1}=k(k \geq 1)$ during $[0, T)$ and aviation ammunition equipment maintenance materials consumption when condition-based replacement is given at $T$ time is

$$
\begin{gathered}
P\left(\sum_{i=1}^{k} T_{i}<T-T_{0}, y_{1}=k\right) \\
=P\left(\sum_{i=1}^{k} T_{i}<T-T_{0}, T_{k+1}>T-\sum_{i=1}^{k} T_{i}\right) \\
=\int_{0}^{T-T_{0}} \int_{\substack{k-\sum_{i=1} t_{i} \\
\sum_{i=1}}}^{+\infty} f_{\sum_{i}}\left(\sum_{i=1}^{k} t_{i}\right) f_{T_{k+1}}\left(t_{k+1}\right) \mathrm{d}\left(\sum_{i=1}^{k} t_{i}\right) \mathrm{d} t_{k+1}
\end{gathered}
$$

The probability for aviation ammunition equipment maintenance materials consumption $y_{1}=0$ during $[0, T)$ and aviation ammunition equipment maintenance materials consumption when condition-based replacement is given at $T$ time is

$$
P\left(T_{1}>T, y_{1}=0\right)=P\left(T_{1}>T\right)
$$

The probability for the aviation ammunition equipment maintenance materials consumption $y_{1}=k$ $(k \geq 1)$ during time $[0, T)$ and the aviation ammunition equipment maintenance materials consumption when condition-based replacement is given at $T$ time is

$$
\begin{aligned}
& P\left(\sum_{i=1}^{k} T_{i}<T-T_{0}, y_{1}=k\right) \\
& =P\left(\sum_{i=1}^{k} T_{i}<T-T_{0} \mid y_{1}=k\right) P\left(y_{1}=k\right)
\end{aligned}
$$

So if the aviation ammunition equipment maintenance materials consumption $y_{1}=k \quad(k \geq 1)$ during time $[0, T)$, the probability for aviation ammunition equipment maintenance materials consumption when condition-based replacement is given at $T$ time is

$$
\begin{gathered}
P\left(\sum_{i=1}^{k} T_{i}<T-T_{0} \mid y_{1}=k\right) \\
=\frac{P\left(\sum_{i=1}^{k} T_{i}<T-T_{0}, y_{1}=k\right)}{P\left(y_{1}=k\right)} \\
=\frac{\int_{0}^{T-T_{0}} \int_{\substack{\sum_{i=1}^{k} t_{i} \\
\text { in }}}^{+\infty} f_{\sum_{i=1}^{k} T_{i}}\left(\sum_{i=1}^{k} t_{i}\right) f_{T_{k+1}}\left(t_{k+1}\right) \mathrm{d}\left(\sum_{i=1}^{k} t_{i}\right) \mathrm{d} t_{k+1}}{P\left(\sum_{i=1}^{k} T_{i}<T\right)-P\left(\sum_{i=1}^{k+1} T_{i}<T\right)}
\end{gathered}
$$

If the aviation ammunition equipment maintenance materials consumption $y_{1}=0$ during time period $[0, T)$, the probability for aviation ammunition equipment maintenance materials consumption caused by condition-based maintenance at $T$ time is 1 .

If the aviation ammunition equipment maintenance materials consumption $y_{1}=k(k \geq 1)$ during time period $[0, T)$, the probability for no aviation ammunition equipment maintenance materials consumption when condition-based replacement is given at $T$ time is

$$
\begin{array}{r}
P\left(\sum_{i=1}^{k} T_{i}>T-T_{0} \mid y_{1}=k\right) \\
\int_{0}^{T-T_{0}} \int_{T_{T-\sum_{i=1}^{k} t_{i}}^{+\infty} f_{\sum_{i=1} T_{i}}\left(\sum_{i=1}^{k} t_{i}\right) f_{T_{k+1}}\left(t_{k+1}\right) \mathrm{d}\left(\sum_{i=1}^{k} t_{i}\right) \mathrm{d} t_{k+1}}^{P\left(\sum_{i=1}^{k} T_{i}<T\right)-P\left(\sum_{i=1}^{k+1} T_{i}<T\right)}
\end{array}
$$


If the aviation ammunition equipment maintenance materials consumption $y_{1}=0$ during time $[0, T)$, the probability for no aviation ammunition equipment maintenance materials consumption when conditionbased replacement is given at $T$ time is 0 .

The total probability of aviation ammunition equipment maintenance materials consumption caused by condition-based maintenance at $T$ time can be obtained by summing probability of aviation ammunition equipment maintenance materials consumption $y_{1}=k$ ( $k \geq 0)$ during $[0, T)$ and the probability of aviation ammunition equipment maintenance materials consumption caused by condition-based maintenance at $T$ time:

$$
\begin{aligned}
& P\left(T_{1}>T\right)+\sum_{k=1}^{+\infty} P\left(\sum_{i=1}^{k} T_{i}<T-T_{0}, y_{1}=k\right) \\
& =\int_{T}^{+\infty} f_{T_{1}}\left(t_{1}\right) \mathrm{d} t_{1}+\sum_{k=1}^{+\infty}\left[\int_{0}^{T-T_{0}} \int_{\substack{T_{0} \\
T-\sum_{i=1} t_{i}}}^{+\infty} f_{\sum_{i=1} T_{i}}\left(\sum_{i=1}^{k} t_{i}\right) f_{T_{k+1}}\left(t_{k+1}\right) \mathrm{d}\left(\sum_{i=1}^{k} t_{i}\right) \mathrm{d} t_{k+1}\right]
\end{aligned}
$$

The total probability for aviation ammunition equipment maintenance materials consumption caused by condition-based maintenance at $T$ time can also be described as the average value of aviation ammunition equipment maintenance materials consumption at $T$ time.

\section{APPLICATIONS}

We Suppose a certain organization possesses one pieces of equipment, and each piece of equipment is maintained by the combination of periodic maintenance and corrective maintenance. During the normal operation, the service life of a certain unit obeys the gamma distribution, of which the shape parameter is $\alpha_{1}=5$, the scale parameter is $\beta_{1}=0.02 / \mathrm{h}$. Under condition-based maintenance, if the reliability of a certain unit falls below the rated value $w=0.5$, replacing maintenance will be given, otherwise no replacement will be given; if the equipment fault occurs during the normal operation, an immediate replacement of the units should be given. Through the analysis of the aviation ammunition equipment maintenance materials consumption rule of one unit within $[0,5000 \mathrm{~h}]$, we can predict the spare part consumption amount of all equipment within [0,5000h] and determine the stock amount of aviation ammunition equipment maintenance materials.

So the probability for aviation ammunition equipment maintenance materials consumption $y_{1}=k$ $(k=1,2,3,4 \cdots)$ within $[0,5000 \mathrm{~h}]$ is

$$
P\left(y_{1}=k\right)=\int_{0}^{5000} \frac{0.02^{5 k}}{\Gamma(5 k)} t^{5 k-1} \mathrm{e}^{-0.02 t} \mathrm{~d} t-\int_{0}^{5000} \frac{0.02^{5(k+1)}}{\Gamma[5(k+1)]} t^{5(k+1)-1} \mathrm{e}^{-0.02 t} \mathrm{~d} t
$$

The probability for aviation ammunition equipment maintenance materials consumption $y_{1}=0$ within $[0,5000 \mathrm{~h}]$ is

$$
P\left(y_{1}=0\right)=1-\int_{0}^{5000} \frac{0.02^{5}}{\Gamma(5)} t^{5-1} \mathrm{e}^{-0.02 t} \mathrm{~d} t
$$

The distribution of aviation ammunition equipment maintenance materials consumption is shown in Fig. 1.

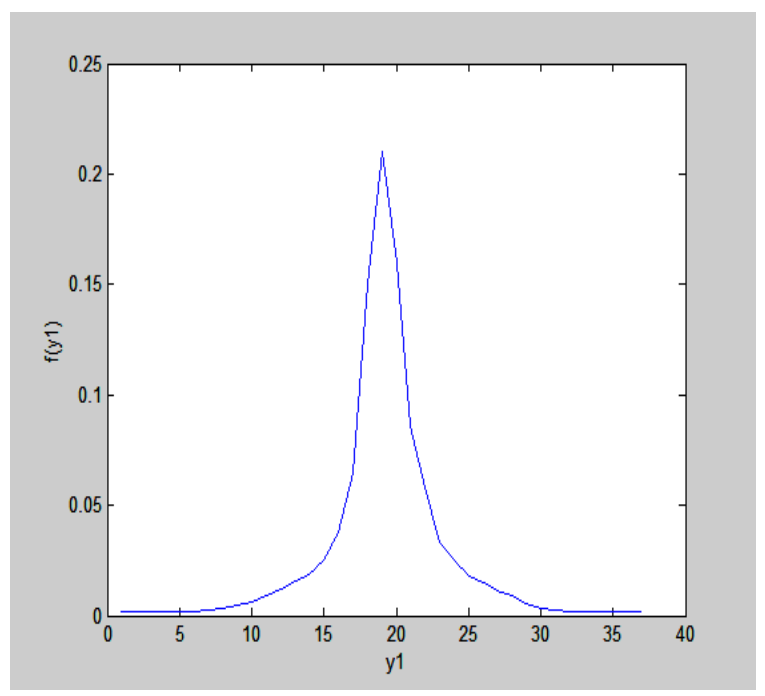

Fig. 1 Distribution of Materials Consumption

The average aviation ammunition equipment maintenance materials consumption is

$$
\bar{y}_{1}=\sum_{k=1}^{+\infty} \int_{0}^{5000} \frac{0.02^{5 k}}{\Gamma(5 k)} t^{5 k-1} \mathrm{e}^{-0.02 t} \mathrm{~d} t
$$

Using MATLAB programming software, we can obtain $\bar{y}_{1}=20.3$.

By using the models established in this paper, the accurate prediction value of consumption amount of condition-based replacement units can be obtained, leading to a more scientific formulation of stock amount of condition-based replacement units, which can further increase the military economic benefit of aviation ammunition equipment maintenance materials support.

\section{CONCLUSIONS}

Nearly all the segments about aviation ammunition equipment maintenance materials include acquisition, storage, supplying and management have close connections with the aviation ammunition equipment maintenance materials consumption information.

The application of the aviation ammunition equipment maintenance materials consumption models based on the reliability of the units under conditionbased maintenance could be extended and the aviation ammunition equipment maintenance materials consumption models could also be improved aiming at solving different problems.

\section{ACKNOWLEDGEMENTS}

This work is supported by the Education Sciencefund of the Education Department of Jiangsu, China (No.2013JGA127). 


\section{REFERENCES}

[1] Ni Xiancun, Zuo Hongfu, Chen Feng teng and Rong Xiang, "Civil Aircraft Rotable Spare Parts Forecasting”, Journal of Nanjing University of Aeronautics \& Astronautics, vol. 41, pp. 253-256, Apr. 2009.

[2] LI Dawei, Zhang Zhihua and Liu Tianhua, "A regulated method of initial spare based on the Bayes method", Systems Engineering-Theory \& Practice, vol. 33, pp. 2967-2971, Nov. 2013.

[3] Zhao Jianzhong, Xu Tingxue, Liu Yong and Yin Yantao, "Consumption Forecasting of Missile Spare Parts Based on Rough Set, Entropy Weight and Improved SVM", Acta Armamentarii, vol. 33, pp. 1258-1265, Oct. 2012. 